\title{
ORIGINAL
}

\section{Early application of airway pressure release ventilation may reduce the duration of mechanical ventilation in acute respiratory distress syndrome}

\author{
Yongfang Zhou, Xiaodong Jin, Yinxia Lv, Peng Wang, Yunqing Yang, Guopeng Liang, Bo Wang and Yan Kang * (D)
}

(c) 2017 The Author(s). This article is an open access publication

\begin{abstract}
Purpose: Experimental animal models of acute respiratory distress syndrome (ARDS) have shown that the updated airway pressure release ventilation (APRV) methodologies may significantly improve oxygenation, maximize lung recruitment, and attenuate lung injury, without circulatory depression. This led us to hypothesize that early application of APRV in patients with ARDS would allow pulmonary function to recover faster and would reduce the duration of mechanical ventilation as compared with low tidal volume lung protective ventilation (LTV).

Methods: A total of 138 patients with ARDS who received mechanical ventilation for $<48 \mathrm{~h}$ between May 2015 to October 2016 while in the critical care medicine unit (ICU) of the West China Hospital of Sichuan University were enrolled in the study. Patients were randomly assigned to receive APRV $(n=71)$ or LTV $(n=67)$. The settings for APRV were: high airway pressure $\left(\mathrm{P}_{\text {high }}\right)$ set at the last plateau airway pressure $\left(\mathrm{P}_{\text {plat }}\right)$, not to exceed $\left.30 \mathrm{cmH}_{2} \mathrm{O}\right)$ and low airway pressure ( $\mathrm{P}_{\text {low }}$ ) set at $5 \mathrm{cmH}_{2} \mathrm{O}$; the release phase $\left(\mathrm{T}_{\text {low }}\right.$ ) setting adjusted to terminate the peak expiratory flow rate to $\geq 50 \%$; release frequency of 10-14 cycles/min. The settings for LTV were: target tidal volume of $6 \mathrm{~mL} / \mathrm{kg}$ of predicted body weight; $\mathrm{P}_{\text {plat }}$ not exceeding $30 \mathrm{cmH}_{2} \mathrm{O}$; positive end-expiratory pressure (PEEP) guided by the PEEP-FiO 2 table according to the ARDSnet protocol. The primary outcome was the number of days without mechanical ventilation from enrollment to day 28 . The secondary endpoints included oxygenation, $\mathrm{P}_{\text {plat }}$, respiratory system compliance, and patient outcomes.

Results: Compared with the LTV group, patients in the APRV group had a higher median number of ventilator-free days $\{19$ [interquartile range (IQR) 8-22] vs. $2($ IQR $0-15) ; P<0.001\}$. This finding was independent of the coexisting differences in chronic disease. The APRV group had a shorter stay in the ICU $(P=0.003)$. The ICU mortality rate was $19.7 \%$ in the APRV group versus $34.3 \%$ in the LTV group $(P=0.053)$ and was associated with better oxygenation and respiratory system compliance, lower $\mathrm{P}_{\text {plat }}$ and less sedation requirement during the first week following enrollment $(P<0.05$, repeated-measures analysis of variance).
\end{abstract}

Conclusions: Compared with LTV, early application of APRV in patients with ARDS improved oxygenation and respiratory system compliance, decreased $\mathrm{P}_{\text {plat }}$ and reduced the duration of both mechanical ventilation and ICU stay.

\footnotetext{
*Correspondence: Kangyan_5626@hotmail.com

Department of Critical Care Medicine, West China Hospital of Sichuan

University, Chengdu 610041, China
}

Yongfang Zhou and Xiaodong Jin contributed equally to this work.

\section{县


Keywords: Acute respiratory distress syndrome, Airway pressure release ventilation, Low tidal volume, Spontaneous breathing

\section{Introduction}

Although mechanical ventilation is an essential life support for patients with acute respiratory distress syndrome (ARDS), it can cause lung injury due to regional alveolar overstretch and/or repetitive alveolar collapse with shearing (atelectrauma) [1]. Ideally, mechanical ventilation should maintain lung units open throughout the ventilator cycle, which minimizes lung injury due to repetitive alveolar collapse and/or over distention. However, the lung injury may be heterogeneous, with the different lesion areas possibly needing markedly different levels of positive end-expiratory pressure (PEEP) $[2,3]$. In the conventional lung protective ventilation strategy, which combines low tidal volume with sufficient PEEP, the selection of the "optimum" PEEP level to balance the recruitment and over-distension for an individual patient is still an unresolved problem in clinical practice [3-5], and mortality still remains high among those receiving mechanical ventilation [6].

Unlike conventional ventilation which generates tidal volume by raising the airway pressure above the PEEP, airway pressure release ventilation (APRV) delivers a continuous positive airway pressure with a brief intermittent release phase, allowing the release of only partial lung volume and spontaneous breathing throughout the high level [7]. Recent experiments have suggested that compared with the low tidal volume ventilation (LTV), the use of more physiology-driven APRV protocols in animals with ARDS improved alveolar recruitment and gas exchange, increased homogeneity, and reduced lung injury [8-10]. Nonetheless, data on ARDS are limited and usually sourced from small clinical trials in which variable outdated APRV settings have been used to study the use of APRV; consequently, the findings of these studies are controversial [11-15]. As such ARPV remains an unproven therapy for patients with ARDS. We hypothesized that in patients with ARDS, early application of the updated APRV methodology would better improve oxygenation and respiratory system compliance and reduce the duration of mechanical ventilation compared to conventional LTV [4].

\section{Materials and methods \\ Patients}

We performed this trial in the critical care medicine department of West China Hospital of Sichuan University, Sichuan province, China. This study was approved by the ethics committee of West China Hospital of Sichuan
University in accordance with the Helsinki Declaration. Written informed consent was obtained from the patients' authorized surrogates. The clinical trial registration number was NCT02639364.

Patients who met the inclusion criteria were enrolled in the study from May 2015 to October 2016: fulfilled the diagnostic criteria of ARDS, according to the Berlin definition [16]; had a ratio of partial pressure arterial oxygen and fraction of inspired oxygen $\left(\mathrm{PaO}_{2}: \mathrm{FiO}_{2}\right)$ of $\leq 250$ during invasive mechanical ventilation [17]; had received endotracheal intubation and mechanical ventilation for $<48 \mathrm{~h}$ prior to inclusion [17]. The exclusion criteria of the study were as follows: pregnancy; anticipated duration of invasive mechanical ventilation for $<48 \mathrm{~h}$; intracranial hypertension (suspected or confirmed); neuromuscular disorders that are known to prolong the need for mechanical ventilation; severe chronic obstructive pulmonary disease; preexisting conditions with an expected 6-month mortality exceeding 50\%; presence of documented barotrauma; treatment with extracorporeal support (ECMO) at enrollment; refractory shock; lack of commitment to life support; age of $<18$ years or $>85$ years. Eligible patients were randomly assigned to the APRV group or to the LTV group by random selection of opaque sealed envelopes for consecutive patients from a box of 138 envelopes. Each envelope contained a number by a random allocation process using a computer-generated random block design.

\section{Ventilator setting}

Respiratory therapists performed ventilator management. All patients were initially ventilated with volume assisted-control ventilation (VCV) using a Puritan Bennett $^{\mathrm{TM}} 840$ Ventilator (Covidien, Medtronic Inc. Minneapolis, MN) prior to randomization to the APRV study arm or LTV study arm. In both groups, the mechanical ventilation goals were to maintain plateau airway pressure $\left(\mathrm{P}_{\text {plat }}\right)$ at no more than $30 \mathrm{cmH}_{2} \mathrm{O}, \mathrm{PaO}_{2}$ at between 55 and $100 \mathrm{~mm} \mathrm{Hg}$ (or pulse oximeter between 88 and $98 \%)$, and arterial $\mathrm{pH}$ at $\geq 7.30$. $[4,18]$.

\section{LTV group}

In the LTV group, tidal volume target $\left(\mathrm{V}_{\mathrm{T}}\right)$ was $6 \mathrm{~mL} /$ $\mathrm{kg}$ predicted body weight (PBW), with allowances for 4-8 $\mathrm{mL} / \mathrm{kg}$ PBW to minimize asynchrony between the patient and ventilator; PEEP levels were adjusted, guided by the PEEP-FiO 2 table, and then $\mathrm{V}_{\mathrm{T}}$ and the respiratory rate were regulated to achieve the above target $\mathrm{pH}$ 
Table 1 Baseline characteristics of the patients

\begin{tabular}{|c|c|c|}
\hline Patient characteristic & APRV group $(n=71)$ & LTV group $(n=67$ \\
\hline Male sex & $50(70.4 \%)$ & $41(61.2 \%)$ \\
\hline Age (years) & $51.5 \pm 15.0$ & $52.0 \pm 15.1$ \\
\hline Predicted body weight (kg) & $61.7 \pm 8.2$ & $60.5 \pm 7.3$ \\
\hline APACHE II score at admission & $22.0 \pm 7.9$ & $20.2 \pm 7.6$ \\
\hline Duration of mechanical ventilation (h) & $24.6 \pm 12.6$ & $22.1 \pm 13.5$ \\
\hline Duration of ICU stay before inclusion (h) & $25.6 \pm 12.6$ & $23 \pm 13.3$ \\
\hline \multicolumn{3}{|l|}{ Chronic disease } \\
\hline Chronic obstructive pulmonary disease & $2(2.8 \%)$ & $5(7.5 \%)$ \\
\hline Chronic cardiac dysfunction & $2(2.8 \%)$ & $3(4.5 \%)$ \\
\hline Chronic renal dysfunction & $0 \%$ & $3(4.5 \%)$ \\
\hline Hematological disease & $2(2.8 \%)$ & $3(4.5 \%)$ \\
\hline Hepatic disease & $3(4.2 \%)$ & $5(7.5 \%)$ \\
\hline Cancer & $7(9.9 \%)$ & $12(17.9 \%)$ \\
\hline Immunodeficiency & $4(5.6 \%)$ & $4(6.0 \%)$ \\
\hline Diabetes & $3(4.2 \%)$ & $2(3.0 \%)$ \\
\hline Coexisting one or more of the above diseases & $23(32.4 \%)$ & $34(50.7 \%)$ \\
\hline \multicolumn{3}{|l|}{ Reason for ARDS } \\
\hline Pneumonia & $18(25.4 \%)$ & $26(38.8 \%)$ \\
\hline Extrapulmonary sepsis & $13(18.3 \%)$ & $10(14.9 \%)$ \\
\hline Severe acute pancreatitis & $19(26.8 \%)$ & $13(19.4 \%)$ \\
\hline Severe trauma & $9(12.7 \%)$ & $7(10.4 \%)$ \\
\hline Major surgical procedures & $8(11.3 \%)$ & $9(13.4 \%)$ \\
\hline Other & $4(5.6 \%)$ & $2(3.0 \%)$ \\
\hline \multicolumn{3}{|l|}{ Arterial blood gases at baseline } \\
\hline $\mathrm{pH}$ & $7.37 \pm 0.09$ & $7.38 \pm 0.10$ \\
\hline $\mathrm{PaCO}_{2}(\mathrm{mmHg})$ & $40.1 \pm 7.4$ & $41.7 \pm 10.5$ \\
\hline $\mathrm{FiO}_{2}$ & $0.66 \pm 0.19$ & $0.62 \pm 0.19$ \\
\hline $\mathrm{PaO}_{2}(\mathrm{~mm} \mathrm{Hg})$ & $72.5 \pm 13.1$ & $76.8 \pm 20.5$ \\
\hline $\mathrm{PaO}_{2}: \mathrm{FiO}_{2}$ at baseline & $121.7 \pm 46.8$ & $138.3 \pm 56.1$ \\
\hline $\mathrm{PaO}_{2}: \mathrm{FiO}_{2} \leq 150$ & $47(66.2 \%)$ & $41(61.2 \%)$ \\
\hline \multicolumn{3}{|l|}{ Co-interventions } \\
\hline Vasopressor & $40(56.3 \%)$ & $46(68.7 \%)$ \\
\hline
\end{tabular}

Data are presented as the mean \pm standard deviation (SD), or as a number with the percentage in parenthesis, as appropriate (\%)

APRV Airway pressure release ventilation, LTV low tidal volume lung protective ventilation (ARDSnet protocol), APACHEII Acute Physiology and Chronic Health Evaluation II, ICU intensive care unit, $A R D S$ acute respiratory distress syndrome, $\mathrm{PaCO}_{2}$ partial pressure of arterial carbon dioxide, $\mathrm{PaO} \mathrm{O}_{2}$ partial pressure of arterial oxygen, $\mathrm{FiO}_{2}$ fraction of inspired oxygen,

and $\mathrm{P}_{\text {plat }}$ values according to the ARDSnet protocol [4, 19]. In the setting of hypotension (mean arterial pressure of $<60 \mathrm{~mm} \mathrm{Hg}$ ) or pneumothorax occurrence, PEEP levels were allowed to be further modified, according to the individual patient's needs; if the $\mathrm{PaO}_{2}: \mathrm{FiO}_{2}$ ratio was $<150$ with $\mathrm{FiO}_{2}>0.6$, PEEP levels could be further titrated by the ways of optimum respiratory compliance or oxygenation, at the clinician's discretion. If the patient presented severe respiratory acidosis $(\mathrm{pH}<7.15)$, the respiratory rate was increased to 35 breaths per minute, with titrations made in $\mathrm{V}_{\mathrm{T}}\left(\mathrm{P}_{\text {plat }}\right.$ target of $30 \mathrm{cmH}_{2} 0$ may be exceeded), according to the ARDSnet protocol
[4]. If severe respiratory acidosis persisted $(\mathrm{pH}<7.15)$, $\mathrm{NaHCO}_{3}$ could be given [Appendix in Electronic Supplementary Material (ESM)].

\section{APRV group}

Patients were transitioned from their previous volume assist-controlled ventilation to APRV with the following initial settings: high airway pressure $\left(\mathrm{P}_{\text {high }}\right)$ was set at the $\mathrm{P}_{\text {plat }}$ measured at the previous VCV settings, not to exceed $30 \mathrm{~cm} \mathrm{H}_{2} \mathrm{O}$; low airway pressure $\left(\mathrm{P}_{\text {low }}\right)$ was set at $5 \mathrm{cmH}_{2} \mathrm{O}$ (minimal pressure level was used to prevent atelectasis per standard practice); duration of release 
phase $\left(\mathrm{T}_{\text {low }}\right)$ was initially set at one- to 1.5 -fold the expiratory time constant, and then adjusted to achieve a termination of peak expiratory flow rate (PEFR) of $\geq 50 \%$ of PEFR; release frequency was $10-14$ frequency/min; duration of $\mathrm{P}_{\text {high }}\left(\mathrm{T}_{\text {high }}\right)$ was indirectly calculated based on the $\mathrm{T}_{\text {low }}$ and release frequency $[9,20]$; initially spontaneous respiratory level was targeted as spontaneous minute ventilation $\left(\mathrm{MV}_{\text {spont }}\right)$, approximately $30 \%$ total minute ventilation $\left(M V_{\text {total }}\right)$ (for details on the APRV settings for titration, see ESM Appendix Tables 3-5).

\section{Analgesia and sedation}

In the both groups, analgesia and sedation were managed to achieve the desired level of analgesia and sedation. The analgesia target level was a Critical-Care Pain Observational Tool (CPOT) score of $0-2$, and the sedation goal was a Richmond Agitation Sedation Scale (RASS) score of -2 to 0 . If patients exhibited anxiety, agitation, and/ orrespiratory distress, or they fought the ventilator, they would receive deeper sedation at less than a RASS score of -2 . According to our local sedation procedure, RASS and CPOT scores were assessed and recorded every $4 \mathrm{~h}$ (or more frequently when indicated) by the nursing staff, who adjusted the dosages of analgesic and sedative drugs to maintain the analgesia and sedation target level. In the APRV group, respiratory therapists would further titrate APRV settings and dosages of analgesics and sedatives to achieve the target level of spontaneous breathing level [21] (for details, see ESM Appendix Tables 3-5).

\section{Procedures in both groups}

For patients with severe hypoxemia (with no response to the assigned protocol and $\mathrm{PaO}_{2}: \mathrm{FiO}_{2}$ ratio of $<100$ during invasive mechanical ventilation for at least $12 \mathrm{~h}$ ), clinicians could apply other supportive therapies for hypoxemia (e.g., recruitment maneuvers, prone positioning, neuromuscular blockade, or inhalation nitric oxide) in both groups (see ESM Appendix for details). Patients could receive rescue measures (including high frequency oscillatory ventilation or ECMO) at the clinician's discretion, in case of any one of the following life-threatening events: refractory hypoxemia $\left(\mathrm{PaO}_{2}<55 \mathrm{~mm} \mathrm{Hg}\right.$ with an $\mathrm{FiO}_{2}$ of 1.0), refractory barotrauma (chest tube with active air leak, persistent pneumothorax, and/or subcutaneous emphysema despite pleural space drainage), refractory respiratory acidosis ( $\mathrm{pH}$ of $\leq 7.15$ ), or refractory shock (even if sufficient fluid resuscitation and usage of vasoactive drugs).

Additionally, physicians applied usual care interventions for the general management of critically ill patients, according to the current guideline standards. Starting the first day following enrollment, in the LTV group if patients received deeper sedation (RASS score of $<-2$ ), the physicians would once daily interrupt the sedation, and the respiratory therapists would manage patients with the Spontaneous Breathing Trials (SBT) safety screen every morning. Those patients who passed the SBT safety screen underwent a 30-min SBT with a pressure support ventilation of $5-7 \mathrm{cmH}_{2} \mathrm{O}$, PEEP of 5 $\mathrm{cmH}_{2} \mathrm{O}$, and $\mathrm{FiO}_{2}$ of $\leq 40 \%$ [21]. In the APRV group, in the first stage, as published previously $[15,20], \mathrm{P}_{\text {high }}$ was gradually reduced by $2 \mathrm{cmH}_{2} \mathrm{O}$, simultaneously with a reduction in release rate by two frequencies/min, twice daily unless the patient's cardiopulmonary function deteriorated. In the second stage, when patients achieved the criteria with a $\mathrm{P}_{\text {high }}$ of $20 \mathrm{cmH}_{2} \mathrm{O}$ on $40 \% \mathrm{FiO}_{2}$, the respiratory therapist immediately started to perform the same weaningprotocol with SBT trial as in the LTV group [17]. When the SBT was successful, physicians and respiratory therapists decided to extubate the patients (for details see ESM Appendix).

The primary endpoint was the number of ventilatorfree days at day 28 (if patients died during the 28-day period after enrollment, the number of ventilator-free days was zero). The secondary endpoints included oxygenation and respiratory mechanics, such as $\mathrm{P}_{\text {plat }}$, mean airway pressure, respiratory system compliance at baseline and on days $1,2,3$, and 7 , as well as clinical outcomes [the length of stay in the intensive care unit (ICU) and hospital, ICU mortality and hospital mortality, and the occurrence of adverse events]. $\left(\mathrm{P}_{\text {plat }}\right.$ and respiratory system compliance measurements during the APRV are detailed in ESM Appendix. Fig. S1).

\section{Statistical analysis}

The primary outcome was the number of ventilatorfree days at day 28 . The mean ( \pm standard deviation) number of ventilator-free days from day 1 to day 28 is $14.5 \pm 10.4$ days in the low tidal volume and lower PEEP group according to Brower and colleagues, the ARDS network [22]. Putensen reported that APRV could shorted the duration of ventilator support by 6 days in patients at risk of ARDS compared with those pressurecontrolled ventilation [11]. We conservatively estimated that a sample size of 110 patients would be required to detect an increment of 5 days in the number ventilatorfree days at day 28 in the APRV group with $80 \%$ power and a two-sided significance level of 0.05 . In total, 138 patients were enrolled in the study in order to manage the drop-outs.

Data are expressed as the mean \pm standard deviation, and as the median and interquartile ranges (IQR), or percentages. Continuous variables with a normal distribution were analyzed with the Student's $t$ test, Continuous variables with non-normal distribution were compared with the use of the Kruskal-Wallis analysis of variance. 
Dichotomous or nominal categorical variables were analyzed by either the Pearson Chi-square or Fisher's exact test. The trend was assessed over time in oxygenation and respiratory mechanics repeated measurements by comparing the LTV group and APRV group at baseline and on days $1,2,3$, and 7 , with the use of the repeated-measures analysis. A two-sided $P$ value of $<0.05$ was considered to indicate statistical significance.

\section{Results}

From May 2015, through to October 2016, a total of 138 patients with ARDS were enrolled in this intentionto-treat analysis: 71 patients in the APRV group and 67 patients in the LTV group (Fig. 1). The proportion of patients with an arterial oxygenation index $\left(\mathrm{PaO}_{2} / \mathrm{FiO}_{2}\right)$ of $<150 \mathrm{mmHg}$ was similar between the APRV and LTV groups ( 66.2 vs. $61.2 \%$, respectively; $P=0.541$ ). Most of the patients in the two groups were severely ill, with a mean APACHE II (Acute Physiology and Chronic Health Evaluation II) score of $22.0 \pm 7.9$ in the APRV group and $20.2 \pm 7.6$ in the LTV group $(P=0.178)$ (Table 1$)$.

\section{Respiratory and hemodynamic parameters and analgesia and sedation variables at baseline and on days 1, 2, 3, and 7 after enrollment}

Respiratory parameters and arterial blood gas measurements at baseline in the APRV group were similar to those in the LTV group, with the exception of respiratory rate which was higher in the APRV group $(P=0.039)$ (Table 2). On the third therapeutic day (Table 2), respiratory system compliance and the $\mathrm{PaO}_{2}: \mathrm{FiO}_{2}$ ratio were significantly improved in the APRV group compared to the LTV group $(P<0.001$, respectively). The ventilation setting frequency was lower in the APRV group $(P=0.002)$, but the monitoring of respiratory rates was similar in both groups. The values of $\mathrm{P}_{\text {peak }}, \mathrm{PEEP}$, and $\mathrm{P}_{\text {plat }}$ were significantly lower in the APRV group $(P<0.01)$, the mean airway pressure was $5.8 \mathrm{cmH}_{2} \mathrm{O}$ higher in the APRV group than in the LTV group $(P<0.001)$, but the driving pressure was similar in both groups. The mean spontaneous minute ventilation was $1.78 \pm 1.37 \mathrm{~L} / \mathrm{min}$ in the APRV group. The total minute ventilation was lower in the APRV group than in the LTV group $(P=0.001)$; however, the values of $\mathrm{PaCO}_{2}$ and $\mathrm{pH}$ were similar in both groups. Heart rate and arterial blood pressure were slightly improved $(P<0.05$, respectively).

On days 1, 2, 3, and 7, compared to the LTV group, the mean airway pressure was higher in the APRV group $(P<0.001$, by repeated-measures analysis of variance) (Fig. 2b) and respiratory system compliance and $\mathrm{PaO}_{2}: \mathrm{FiO}_{2}$ were significantly better in the APRV group $(P=0.003$ by repeated-measures analysis of variance) (Fig. 2c, d). On day 1 , the $\mathrm{PaO}_{2}: \mathrm{FiO}_{2}$ value was $66.3 \mathrm{mmHg}$ higher in the APRV group than in the LTV group $(P<0.001)$. Heart rates were lower and mean arterial pressures were a little higher in the APRV group than in the LTV group on days 2,3 , and $7(P<0.05$ by repeated-measures analysis of variance) (Fig. 2e, f). The average doses of norepinephrine were similar $(P=0.612)$ (Fig. 2g). Compared with the LTV group, the sedation depth in the APRV group was lighter $(P<0.001)$ (Fig. $2 \mathrm{~h}$ ), and the average doses of fentanyl and midazolam were significantly lower $(P<0.01)($ Fig. $2 \mathrm{i}, \mathrm{j})$, and the average doses of propofol were similar $(P=0.112)$ (Fig. $2 \mathrm{k}$ ).

\section{Study Outcomes}

Study outcomes are presented in Table 3. The APRV protocol was associated with an increased number of ventilator-free days on day 28 than the LTV protocol [median 19 days (IQR $8-22$ ) vs. 2 days (IQR $0-15$ ), respectively; $P<0.001$ ] (Table 3 and Fig. 3); this result was supported by the per-protocol analysis results which also showed a similar difference [median 19 days (IQR 11-22) vs. 8 days (IQR 0-16), respectively; $P<0.001$ ] (ESM Appendix Table S5). Although there were more patients with coexisting chronic diseases and lower sedation depth in the APRV group than in the LTV group $(P<0.05)$, only lighter sedation increased ventilator-free days at day 28 , and APRV still significantly increased ventilator-free days at day 28 $(P<0.001)$ according to the multiple linear regression analysis for correction of the coexisting chronic diseases and sedation depth differences (ESM Appendix Table S4). There was a higher rate of successful extubation in the APRV group than in the LTV group (66.2 vs. $38.8 \%$; $P=0.001$ ), and fewer patients underwent tracheostomy in the APRV group $(P=0.013)$. The APRV protocol significantly decreased the length of ICU stay $(P=0.015)$. The ICU and hospital mortality rates and length of hospital stay were similar for both groups. During the course of the study, more patients in the LTV group received neuromuscular blockers, recruitment maneuvers, and prone ventilation than in the APRV group $(P<0.05)$.

\section{Discussion}

The main findings of this study were that compared with the LTV group, there was a shorter duration of mechanical ventilation in the APRV group, and early use of APRV in patients with ARDS could significantly improve oxygenation and respiratory system compliance, decrease plateau airway pressure, and reduce sedation requirement. Patients in the APRV group also had shorter length of stay in the ICU, higher rate of successful extubation, and lower tracheostomy rate than did patients in the LTV group. However, there was no 


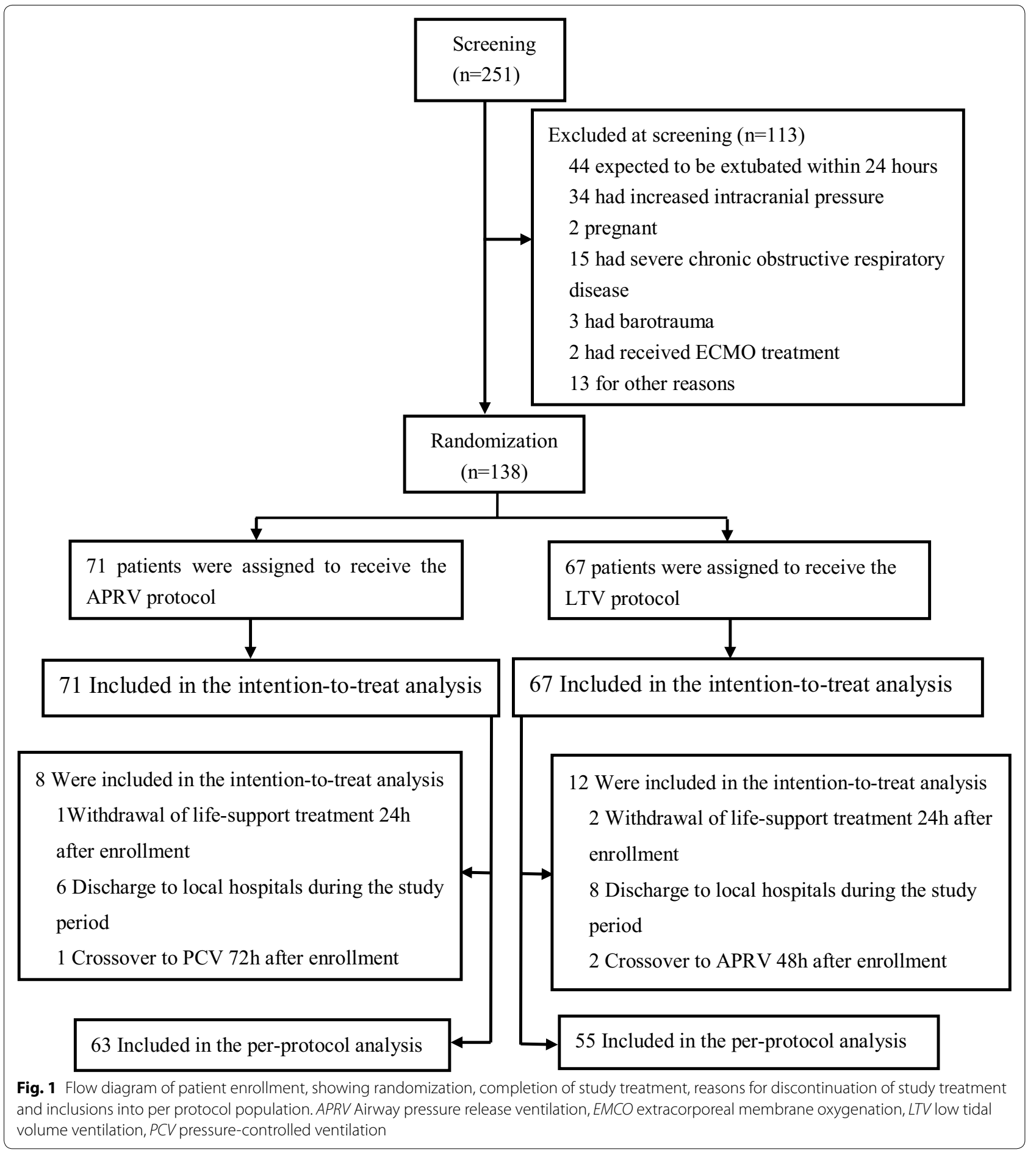

difference in hospital length of stay, ICU mortality, hospital mortality, or incidence of pneumothorax between the two groups.

\section{Respiratory mechanics and gas exchange}

The data of the present study are in agreement with previously reported clinical and experimental findings 
Table 2 Respiratory and hemodynamic variables at baseline and on day 3

\begin{tabular}{|c|c|c|c|c|c|c|}
\hline \multirow[t]{2}{*}{ Variable } & \multicolumn{3}{|l|}{ Baseline } & \multicolumn{3}{|c|}{ Day 3 after enrollment ${ }^{c, d}$} \\
\hline & APRV & LTV & $P$ value & APRV & LTV & $P$ value \\
\hline No. of patients & 71 & 67 & & 62 & 56 & \\
\hline \multicolumn{7}{|l|}{ Respiratory variables } \\
\hline Ventilator setting (tidal volume in mL) & $437.8 \pm 40.6$ & $429.6 \pm 47.5$ & 0.277 & - & $423.8 \pm 51.8$ & \\
\hline Ventilator setting (tidal volume in $\mathrm{mL} / \mathrm{kg}$ of predicted body weight) & $7.2 \pm 0.7$ & $7.1 \pm 0.7$ & 0.534 & - & $7.0 \pm 1$ & \\
\hline Ventilator monitoring (tidal volume in $\mathrm{mL}$ ) & $466.6 \pm 54.9$ & $461.2 \pm 59.7$ & 0.578 & $476.9 \pm 111.3$ & $461.8 \pm 64.1$ & 0.364 \\
\hline $\begin{array}{l}\text { Ventilator monitoring (tidal volume in } \mathrm{mL} / \mathrm{kg} \text { of predicted body } \\
\text { weight) }\end{array}$ & $7.6 \pm 1.1$ & $7.7 \pm 1.3$ & 0.619 & $7.8 \pm 1.9$ & $7.7 \pm 1.1$ & 0.575 \\
\hline Ventilator setting frequency (cycles/min) & $15.1 \pm 4.3$ & $15.1 \pm 3.8$ & 0.977 & $12.7 \pm 1.8$ & $14.9 \pm 4.8$ & 0.002 \\
\hline$P_{\text {high }}$ & - & - & & $24.1 \pm 3.6$ & - & \\
\hline PEEP $\left(\mathrm{cmH}_{2} \mathrm{O}\right)$ & $11.4 \pm 3.0$ & $10.4 \pm 2.6$ & 0.063 & $6.9 \pm 1.8$ & $10.4 \pm 2.8$ & $<0.001$ \\
\hline $\mathrm{FlO}_{2}$ & $0.66 \pm 0.19$ & $0.62 \pm 0.19$ & 0.198 & $0.43 \pm 0.09$ & $0.53 \pm 0.19$ & 0.001 \\
\hline Respiratory rate (cycles/min) & $21.5 \pm 6.6$ & $19.5 \pm 4.6$ & 0.039 & $19.0 \pm 6.0$ & $20.3 \pm 5.1$ & 0.225 \\
\hline Peak inspiratory pressure $\left(\mathrm{cmH}_{2} \mathrm{O}\right)$ & $31.7 \pm 4.5$ & $30.4 \pm 4.0$ & 0.061 & $26.2 \pm 3.6$ & $28.5 \pm 4.8$ & 0.005 \\
\hline Mean airway pressure $\left(\mathrm{cmH}_{2} \mathrm{O}\right)$ & $18.3 \pm 3.9$ & $17.4 \pm 3.5$ & 0.140 & $21.8 \pm 3.5$ & $16.0 \pm 3.3$ & $<0.001$ \\
\hline Plateau pressure $\left(\mathrm{cmH}_{2} \mathrm{O}\right)$ & $26.5 \pm 4.0$ & $25.3 \pm 3.6$ & 0.081 & $19.3 \pm 3.9$ & $23.3 \pm 4.6$ & $<0.001$ \\
\hline Driving pressure $\left(\mathrm{cmH}_{2} \mathrm{O}\right)^{\mathrm{a}}$ & $15.2 \pm 3.6$ & $14.8 \pm 3.4$ & 0.550 & $12.6 \pm 3.5$ & $12.8 \pm 4.1$ & 0.822 \\
\hline Respiratory system compliance $\left(\mathrm{mL} / \mathrm{cmH}_{2} \mathrm{O}\right)$ & $30.1 \pm 7.6$ & $32.6 \pm 7.7$ & 0.058 & $43.7 \pm 11.3$ & $34.1 \pm 8.9$ & $<0.001$ \\
\hline Total minute ventilation $(\mathrm{L} / \mathrm{min})^{\mathrm{b}}$ & $8.37 \pm 2.36$ & $8.42 \pm 1.98$ & 0.905 & $6.86 \pm 2.06$ & $8.22 \pm 2.30$ & 0.001 \\
\hline Spontaneous minute ventilation (L/min) & - & - & & $1.78 \pm 1.37$ & - & \\
\hline $\mathrm{pH}$ & $7.37 \pm 0.09$ & $7.38 \pm 0.10$ & 0.427 & $7.42 \pm 0.05$ & $7.42 \pm 0.07$ & 0.648 \\
\hline $\mathrm{PaCO}_{2}(\mathrm{mmHg})$ & $40.1 \pm 7.4$ & $41.7 \pm 10.5$ & 0.307 & $40.8 \pm 7.3$ & $42.3 \pm 8.6$ & 0.291 \\
\hline $\mathrm{PaO}_{2}(\mathrm{mmHg})$ & $72.5 \pm 13.1$ & $76.8 \pm 20.5$ & 0.149 & $116.2 \pm 28.5$ & $84.8 \pm 20.1$ & $<0.001$ \\
\hline $\mathrm{PaO}_{2} \cdot \mathrm{FiO}_{2}$ & $121.7 \pm 46.8$ & $138.3 \pm 56.1$ & 0.060 & $280.3 \pm 83.9$ & $180.5 \pm 68.6$ & $<0.001$ \\
\hline \multicolumn{7}{|l|}{ Hemodynamic variables } \\
\hline Heart rate (beats/min) & $105.4 \pm 22.5$ & $110.2 \pm 24.6$ & 0.238 & $92.7 \pm 16.6$ & $103.6 \pm 19.3$ & 0.001 \\
\hline Systolic blood pressure (mmHg) & $122.2 \pm 17.9$ & $116.2 \pm 22.5$ & 0.088 & $126.6 \pm 18.0$ & $125.0 \pm 20.3$ & 0.646 \\
\hline Diastolic blood pressure $(\mathrm{mmHg})$ & $72.8 \pm 13.2$ & $68.6 \pm 12.1$ & 0.053 & $76.1 \pm 14.5$ & $69.3 \pm 13.3$ & 0.009 \\
\hline Mean arterial pressure $(\mathrm{mmHg})$ & $87.4 \pm 14.7$ & $84.2 \pm 13.4$ & 0.194 & $92.8 \pm 14.9$ & $87.1 \pm 13.6$ & 0.032 \\
\hline
\end{tabular}

Data are presented as the mean \pm SD of the values recorded from 7 am to 12 am on days 1, 2, 3, and 7 after enrollment in each treatment group

$P_{\text {high }}$ High airway pressure

a Driving pressure was calculated as the plateau pressure $\left(\mathrm{P}_{\text {plat }}\right)$ minus positive end expiratory pressure (PEEP)

b Total minute ventilation $=$ release minute ventilation + spontaneous minute ventilation

c Four patients were extubated at day 3, six patients died, three patients withdrew life-support treatment $24 \mathrm{~h}$ after enrollment, seven patients were discharged to their local hospitals; thus, the respiratory and hemodynamic values are given for the 62 ventilated patients in the APRV group and 56 patients in the LTV group

${ }^{d}$ Respiratory system compliance and plateau pressure were monitored by the ventilator (In the APRV group, APRV was temporarily changed to the volumecontrolled ventilation, PEEP was set at the previous monitoring PEEP, tidal volume was set at the previous release volume)

[8-11, 21, 23], namely, that the early use of this APRV protocol in patients with ARDS significantly decreased plateau airway pressure, elevated mean airway pressure, and improved oxygenation and respiratory system compliance, in comparison with LTV ventilation. Furthermore, there was no difference in $\mathrm{PaCO}_{2}$ and $\mathrm{pH}$ between the groups, despite APRV with lower minute ventilation, which indirectly indicated APRV decreasing dead space ventilation.

However, at present, data are only available from a limited number of small randomized prospective human studies with different APRV settings, with some studies showing benefits of APRV on pulmonary function and others showing similarities, as compared with CPPV or LTV $[8,11,12,15]$. For example, one small randomized prospective trial [15] showed that adult trauma patients with acute respiratory failure on APRV or LTV had similar physiological parameters. However, the APRV methodology used in that study was outdated: the upper limit of $\mathrm{P}_{\text {high }}$ was $40 \mathrm{cmH}_{2} \mathrm{O}$, while current evidence suggests that inspiratory-end pressure should be limited to $30 \mathrm{cmH}_{2} \mathrm{O}$ [4]; the $\mathrm{T}_{\text {low }}$ was set at $25-75 \%$ of the PEFR, while $\mathrm{T}_{\text {low }}$ of $<50 \%$ of PEFR could result in dynamic heterogeneity between inspiration and expiration [10]. 

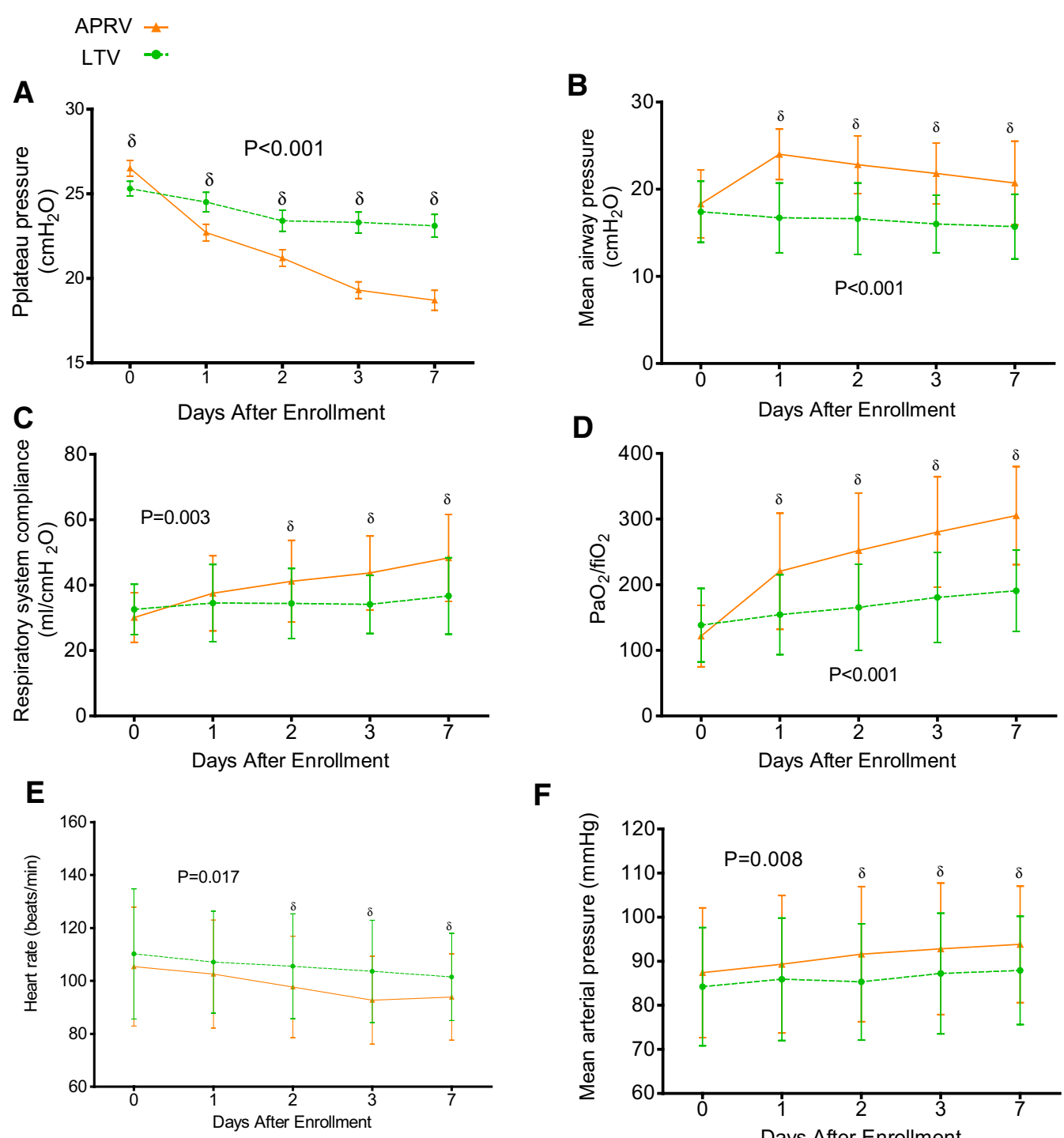

$\mathbf{F}$
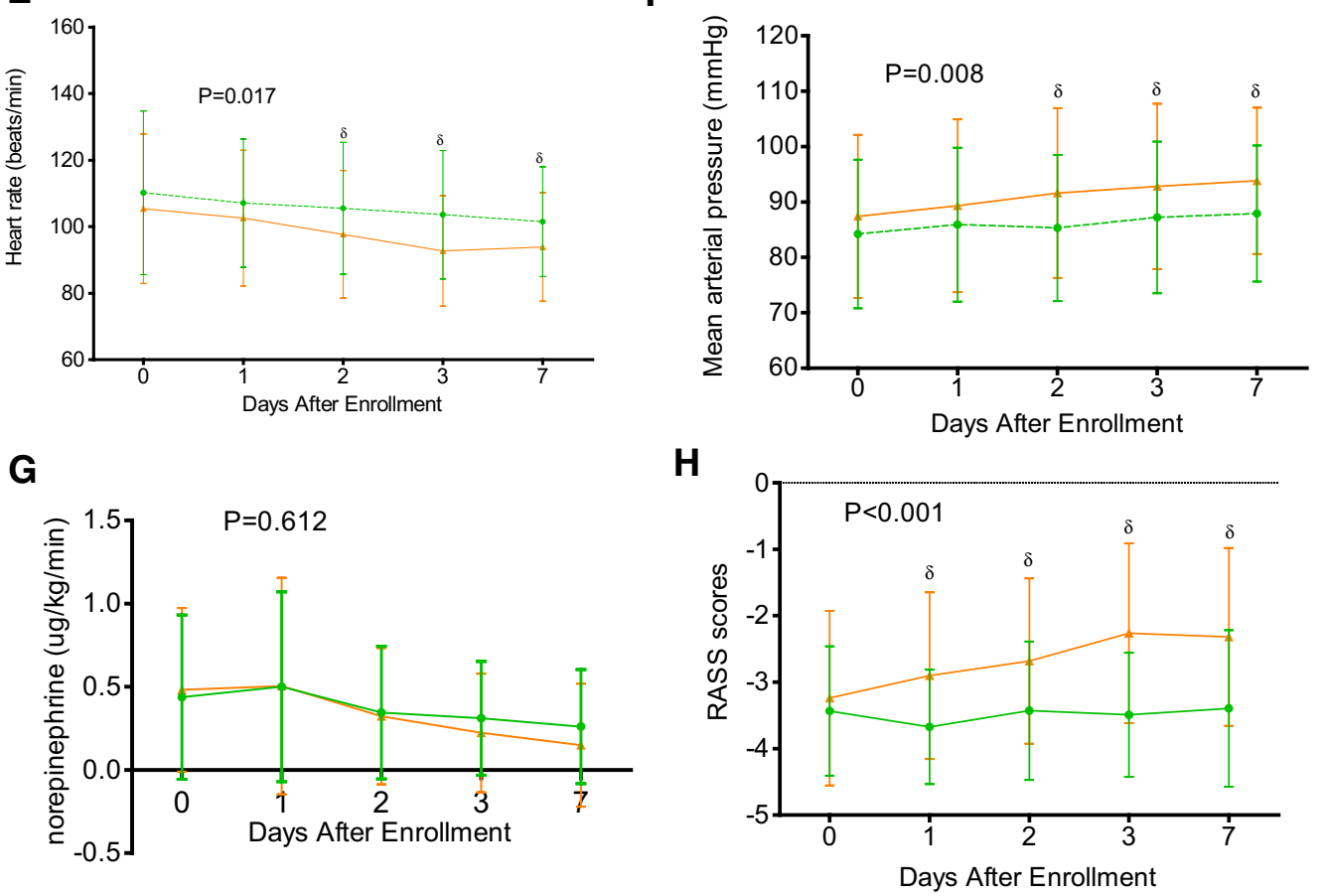

Fig. 2 Respiratory and hemodynamic parameters, and analgesia and sedation variables at baseline and on days 1, 2, 3, and 7 after enrollment. Data are presented as the mean (filled symbols) and standard errors (whiskers). P values were calculated by repeated-measures analysis of variance. a Plateau pressure, $\mathbf{b}$ mean airway pressure, $\mathbf{c}$ respiratory system compliance, $\mathbf{d}$ ratio of the partial pressure of arterial oxygen to the fraction of inspired oxygen $\left(\mathrm{PaO}_{2}: \mathrm{FiO}_{2}\right)$, e heart rate, $\mathbf{f}$ mean arterial pressure, $\mathbf{g}$ average doses of norepinephrine, $\mathbf{h}$ Richmond Agitation Sedation Scale (RASS) scores, $\mathbf{i}$ average doses of fentanyl, $\mathbf{j}$ average doses of midazolam, $\mathbf{k}$ average doses of propofol. All parameters and variables were compared between the two groups at baseline and on days 1, 2, 3, and 7 after enrollment with the Student's $t$ test. Delta denotes that the two-sided $P$ value was $<0.05$ 

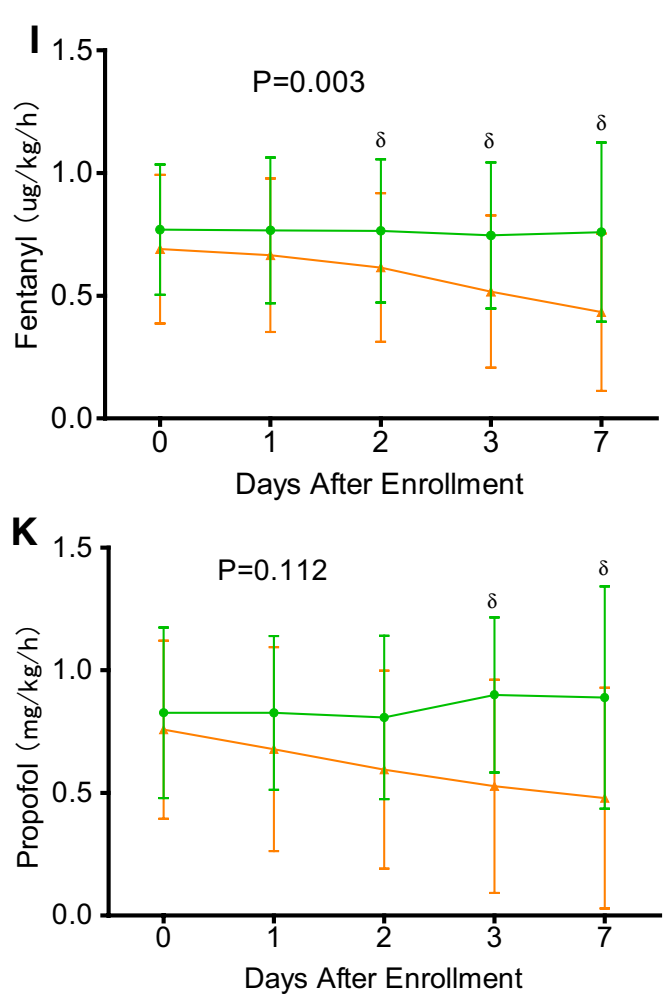

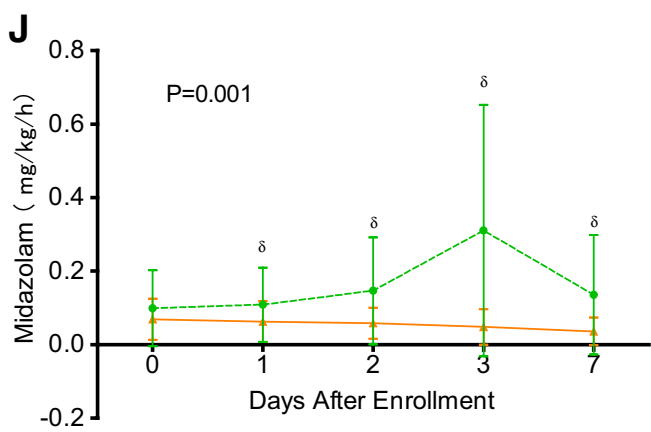

$-0.2$

Fig. 2 continued

According to recent experimental findings, we set the $\mathrm{P}_{\text {high }}$ not to exceed $30 \mathrm{cmH}_{2} \mathrm{O}$ and the $\mathrm{T}_{\text {low }}$ to be at $\geq 50 \%$ of PEFR; these settings were combined with APRV settings and sedation titration to achieve the spontaneous breath target level.

There are collateral channels of ventilation, such as pores of Kohn, which might be additional pathways to facilitate recruitment and redistribute alveolar volume (pressure) throughout the lung over time [24]. The results of previous studies indicate that the process of recruitment and decruitment of lung units should be determined not only by pressure but also by time [25]. For heterogeneous lung injury, during APRV ventilation, the proper elevated baseline airway pressure $\left(\mathrm{P}_{\text {high }}\right)$ and prolonged duration of $\mathrm{P}_{\text {high }}$ would optimize the recruitment of alveoli gradually over time, while prevention of overinflation, and brief release phase $\left(\mathrm{T}_{\text {low }}\right)$ could permit only partial lung volume loss at the release phase, avoid cyclic alveoli collapse, and provide dynamic homogeneity [10]. Recent animal experiments [8-10, 21, 23] have also documented that the updated APRV methodologies attenuate lung injury, preserve surfactant protein and lung architecture, and improve oxygenation, resulting in dynamic alveolar homogeneity without any increase in lung stress and strain.
Additionally, during APRV, allowing moderate spontaneous breath level at the $\mathrm{P}_{\text {high }}$ phase (providing sufficient PEEP) favored lung recruitment and improved ventilation/perfusion matching and lung homogeneous aeration, while minimizing pendelluft and its associated injury [20, 26-28].

\section{Hemodynamics}

The hemodynamic performance and sedation requirement of the patients on APRV in this study are in accordance with previous study findings [11, 29, 30]. APRV favored hemodynamic improvement and reduced sedative and paralysis use, despite the higher mean airway pressure. Ventilation with APRV permitting spontaneous breaths decreased the intrathoracic pressure, thus improving systematic venous return and cardiovascular performance and reducing sedation requirement and the need for paralysis, which may decrease the cardiovascular depression caused by elevated airway pressures $[10$, $20,30]$.

\section{Study outcomes}

This study showed that the APRV group was associated with more days without mechanical ventilation at day 28 and a shorter ICU stay than the LTV group. This finding 


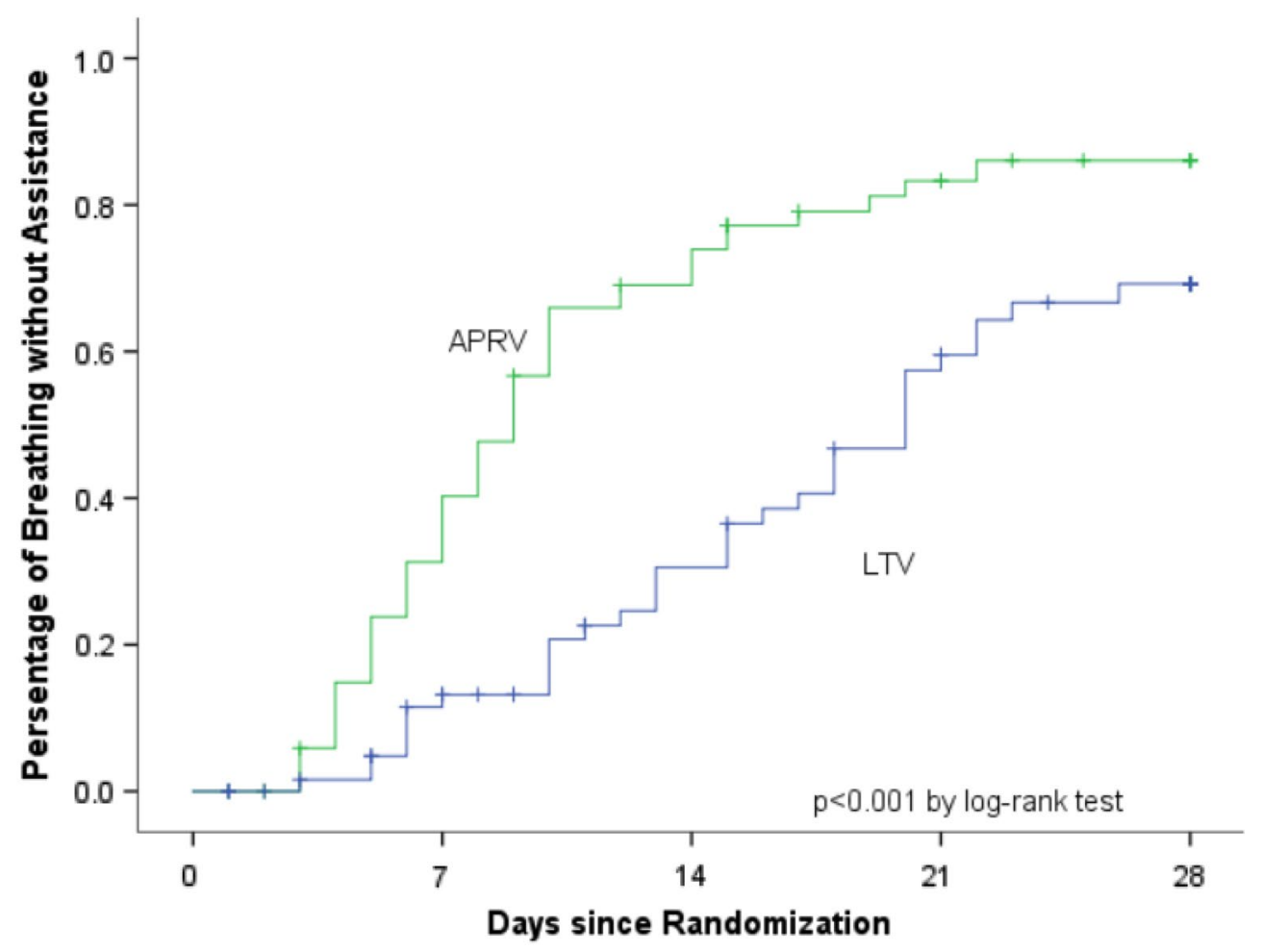

No. at risk

APRV

LTV
71

67
46

52
19

35
8

20
3

12

Fig. 3 Percentage of breathing without assistance from enrollment to day 28 in the APRV and LTV groups

is consistent with previously published results [11], and possible explanations for this finding are as follows. Firstly, early use of APRV improved pulmonary function, such as gas exchange and respiratory compliance. Recent experiments have also documented that early preventative use of APRV can more effectively block ARDS development than LTV $[9,21]$. Putensen et al. reported that the use of APRV in patients with ARDS after $72 \mathrm{~h}$ on pressure-control ventilation improved but did not restore gas exchange and lung mechanics, and prolonged the mechanical ventilation and ICU stay [11]. Secondly, APRV allows moderate spontaneous breathing, reduces sedation and paralysis requirements, and decreases the duration of mechanical ventilation [11, 20, 30, 31]. In the present study, respiratory therapists titrated the APRV settings and dosages of analgesics and sedatives to achieve a moderate spontaneous breath level at the $\mathrm{P}_{\text {high }}$ phase. Our results also show that APRV was associated with lighter sedation, which could increase the number of ventilator-free days at day 28 . Thirdly, in our study there was respiratory therapist-guided weaning protocol with the SBT trial in the LTV group. In the APRV group, in the first stage, to avoid aggressive weaning, the weaning process consisted of simultaneously decreasing both $\mathrm{P}_{\text {high }}$ by $2 \mathrm{cmH}_{2} \mathrm{O}$ and the release rate by two frequencies/min, twice daily unless the patient's cardiopulmonary function deteriorated. In the second stage, when patients achieved the criteria with a $\mathrm{P}_{\text {high }}$ of $20 \mathrm{cmH}_{2} \mathrm{O}$ on $40 \% \mathrm{FiO}_{2}$, respiratory therapists also performed the weaning protocol with the SBT trial as in the LTV group. Two trauma population studies have shown that APRV may increase the number of ventilator days; however, the APRV settings were outdated, and no formal weaning protocol was used $[13,15]$. The current primary APRV weaning process is based on gradual withdrawal, using an alternate decrease in $\mathrm{P}_{\text {high }}$ by $2 \mathrm{cmH}_{2} \mathrm{O}$, followed by an increase in $\mathrm{T}_{\text {high }}$ of $0.5-1.0 \mathrm{~s}$ [15], and extubation is assessed until the criteria of a $\mathrm{P}_{\text {high }}$ of $12 \mathrm{cmH}_{2} \mathrm{O}$ on $40 \% \mathrm{FiO}_{2}$ is achieved [10, 13, $15,20]$. However, evidence suggests that daily SBT can expedite weaning and reduce the duration of mechanical 
Table 3 Main outcome variables

\begin{tabular}{|c|c|c|c|}
\hline Main outcome variables & $\begin{array}{l}\text { APRV } \\
(n=71)^{b}\end{array}$ & $\begin{array}{l}\text { LTV } \\
(n=67)^{b}\end{array}$ & $P$ value \\
\hline No. of days of ventilation & $8[5-14]$ & $15[7-22]$ & 0.001 \\
\hline $\begin{array}{l}\text { No. of ventilator-free days at } \\
28 \text { days }\end{array}$ & 19 [8-22] & $2[0-15]$ & $<0.001$ \\
\hline Successful extubation & $47(66.2 \%)$ & $26(38.8 \%)$ & 0.001 \\
\hline Tracheostomy & $9(12.7 \%)$ & 20 (29.9\%) & 0.013 \\
\hline Length of ICU stay (days) & $15[8-21]$ & $20[10-32]$ & 0.015 \\
\hline $\begin{array}{l}\text { Pneumothorax between day } 1 \\
\text { and day } 28^{\mathrm{a}}\end{array}$ & $3(4.2 \%)$ & $7(10.4 \%)$ & 0.199 \\
\hline Death during the ICU stay & $14(19.7 \%)$ & $23(34.3 \%)$ & 0.053 \\
\hline Length of hospital stay (days) & $21[14-30]$ & $27[18-41]$ & 0.055 \\
\hline Death during the hospital stay & $17(23.9 \%)$ & $25(37.3 \%)$ & 0.088 \\
\hline \multicolumn{4}{|l|}{ Other supportive therapies } \\
\hline Neuromuscular blocker & $2(2.8 \%)$ & $9(13.4 \%)$ & 0.021 \\
\hline Recruitment maneuvers & $4(5.6 \%)$ & $11(16.4 \%)$ & 0.042 \\
\hline Prone position & $2(2.8 \%)$ & $10(14.9 \%)$ & 0.012 \\
\hline Inhaled nitric oxide & $1(1.4 \%)$ & $1(1.5 \%)$ & 1.000 \\
\hline $\begin{array}{l}\text { High-frequency oscillatory } \\
\text { ventilation }\end{array}$ & $1(1.4 \%)$ & $3(4.5 \%)$ & 0.355 \\
\hline
\end{tabular}

Data are expressed as the median with the interquartile range in square brackets for non-normally distributed data or as a number with the percentage in parenthesis for nominal data. The Kruskal-Wallis analysis of variance was used for non-normally distributed data comparisons. Nominal data comparisons were based on either the Chi-squared test or Fisher's exact test

a Two cases of pneumothorax resulted from clinical puncture in the LTV group

b Fourteen patients were discharged to local hospitals and followed up by phone calls. Of these, six patients in the APRV group were discharged to local hospitals, of whom three died, and eight patients in the LTV group were discharged to local hospitals, of whom three died

ventilation as compared with gradually reducing ventilator support [32].

\section{Limitations}

There are several limitations to our study. First, the study was not blinded, as the ventilator settings were obviously different. Secondly, the sample size was small. APRV has evolved into a highly sophisticated, physiology-driven, dynamic mechanical breath profile with precise settings [13], thus a possibility of knowledge bias by the staff was another limitation. However, prior to conducting our study, we first conducted a single-center randomized controlled study and found that all of the respiratory therapists were well trained and skillfully used this study protocol. Thirdly, there were more patients with coexisting chronic diseases in the LTV group than in the APRV group $(P=0.029)$. Using a multivariable analysis for the correction of the coexisting chronic diseases difference, APRV was independent of increasing ventilator-free days at day 28. Finally, in accordance with our APRV protocol, in addition to nursing staff, respiratory therapists were able to further titrate APRV settings and dosages of analgesics and sedatives to achieve the target level of spontaneous breathing. The results of our study show that APRV was associated with lighter sedation, which could increase the number of ventilator-free days at day 28. However, APRV still significantly increased ventilator-free days at day 28 after correcting the sedation difference. Additionally, this study did not measure the patient-ventilator interaction. The questions of whether APRV permitting spontaneous breathing could promote the patient-ventilator synchrony and how the patientventilator dyssynchrony could affect the outcome require further study.

\section{Conclusions}

Compared with conventional LTV, the early application of APRV in patients with ARDS was associated with better oxygenation and respiratory system compliance, lower plateau airway pressure, less sedation requirement, more ventilator-free days at day 28 , and a shorter duration of ICU stay.

\section{Electronic supplementary material \\ The online version of this article (doi:10.1007/s00134-017-4912-z) contains supplementary material, which is available to authorized users.}

\section{Acknowledgements}

The authors acknowledge all the physicians and respiratory therapists in the Department of Critical Care Medicine at West China Hospital of Sichuan University for their participation and assistance in the study.

\section{Author contribution statement}

YFZ, YK and XDJ conceived the trial. YFZ, XDJ, BW, YXL, PW, and YK participated in study design. YFZ, BW, PW, GPL, and YXL recruited patients and collected data, and YFZ, BW, XDJ, GPL, and YK analyzed the data. All authors participated in interpretation of results. YFZ drafted the manuscript, and all authors have reviewed and revised the manuscript. All authors have seen and approved the final version of the manuscript.

\section{Compliance with ethical standards}

\section{Ethics approval and consent to participate}

This study was approved by the ethics committee of West China Hospital of Sichuan University in accordance with the Helsinki Declaration. Written informed consent was obtained from the patients' authorized surrogates. The clinical trial registration number was NCT02639364.

\section{Conflicts of interest}

All authors declare that they do not have any conflict of interest relevant to this study.

\section{Open Access}

This article is distributed under the terms of the Creative Commons Attribution-NonCommercial 4.0 International License (http://creativecommons.org/ licenses/by-nc/4.0/), which permits any noncommercial use, distribution, and reproduction in any medium, provided you give appropriate credit to the original author(s) and the source, provide a link to the Creative Commons license, and indicate if changes were made.

Received: 22 February 2017 Accepted: 12 August 2017

Published online: 22 September 2017 


\section{References}

1. Slutsky AS (1999) Lung injury caused by mechanical ventilation. Chest 116[1 Suppl]:9S-15S

2. Pelosi P, Goldner M, McKibben A, Adams A, Eccher G, Caironi P, Losappio S, Gattinoni L, Marini JJ (2001) Recruitment and derecruitment during acute respiratory failure: an experimental study. Am J Respir Crit Care Med 164(1):122-130

3. Cressoni M, Chiumello D, Algieri I, Brioni M, Chiurazzi C, Colombo A, Colombo A, Crimella F, Guanziroli M, Tomic I, Tonetti T, Luca Vergani G, Carlesso E, Gasparovic V, Gattinoni L (2017) Opening pressures and atelectrauma in acute respiratory distress syndrome. Intensive Care Med 43(5):603-611

4. The Acute Respiratory Distress Syndrome Network (2000) Ventilation with lower tidal volumes as compared with traditional tidal volumes for acute lung injury and the acute respiratory distress syndrome. N Engl J Med 342:1301-1308

5. Terragni PP, Rosboch G, Tealdi A, Corno E, Menaldo E, Davini O, Gandini G, Herrmann P, Mascia L, Quintel M, Slutsky AS, Gattinoni L, Ranieri VM (2007) Tidal hyperinflation during low tidal volume ventilation in acute respiratory distress syndrome. Am J Respir Crit Care Med 175(2):160-166

6. Bellani G, Laffey JG, Pham T, Fan E, Brochard L, Esteban A, Gattinoni L, van Haren F, Larsson A, McAuley DF, Ranieri M, Rubenfeld G, Thompson BT, Wrigge H, Slutsky AS, Pesenti A, LUNG SAFE Investigators; ESICM Trials Group (2016) Epidemiology, patterns of care, and mortality for patients with acute respiratory distress syndrome in intensive care units in 50 countries. JAMA 315(8):788-800

7. Downs JB, Stock MC (1987) Airway pressure release ventilation: a new concept in ventilatory support. Crit Care Med 15:459-461

8. Jain SV, Kollisch-Singule M, Sadowitz B, Dombert L, Satalin J, Andrews P, Gatto LA, Nieman GF, Habashi NM (2016) The 30-year evolution of airway pressure release ventilation (APRV). Intensive Care Med Exp 4(1):11

9. Roy SK, Emr B, Sadowitz B, Gatto LA, Ghosh A, Satalin JM, Snyder KP, Ge L, Wang G, Marx W, Dean D, Andrews P, Singh A, Scalea T, Habashi N, Nieman GF (2013) Preemptive application of airway pressure release ventilation prevents development of acute respiratory distress syndrome in a rat traumatic hemorrhagic shock model. Shock 40:210-216

10. Kollisch-Singule M, Jain S, Andrews P, Smith BJ, Hamlington-Smith KL, Roy S, DiStefano D, Nuss E, Satalin J, Meng Q, Marx W, Bates JH, Gatto LA, Nieman GF, Habashi NM (2016) Effect of airway pressure release ventilation on dynamic alveolar heterogeneity. JAMA 151(1):64-72

11. Putensen C, Zech S, Wrigge H, Zinserling J, Stuber F, Von Spiegel T, Mutz N (2001) Long-term effects of spontaneous breathing during ventilatory support in patients with acute lung injury. Am J Respir Crit Care Med 164:43-49

12. Yoshida T, Rinka H, Kaji A, Yoshimoto A, Arimoto H, Miyaichi T, Kan M (2009) The impact of spontaneous ventilation on distribution of lung aeration in patients with acute respiratory distress syndrome: airway pressure release ventilation versus pressure support ventilation. Anesth Analg 109:1892-1900

13. Maung AA, Schuster KM, Kaplan LJ, Ditillo MF, Piper GL, Maerz LL, Lui FY, Johnson DC, Davis KA (2012) Compared to conventional ventilation, airway pressure release ventilation may increase ventilator days in trauma patients. J Trauma Acute Care Surg 73(2):507-510

14. Bein T, Grasso S, Moerer O, Quintel M, Guerin C, Deja M, Brondani A, Mehta S (2016) The standard of care of patients with ARDS: ventilatory settings and rescue therapies forrefractory hypoxemia. Intensive Care Med 42(5):699-711

15. Maxwell RA, Green JM, Waldrop J, Dart BW, Smith PW, Brooks D, Lewis $P L$, Barker DE (2010) A randomized prospective trial of airway pressure release ventilation and low tidal volume ventilation in adult trauma patients with acute respiratory failure. J Trauma 69:501-510
16. Definition Task Force ARDS, Ranieri VM, Rubenfeld GD, Thompson BT, Ferguson ND, Caldwell E, Fan E, Camporota L, Slutsky AS (2012) Acute respiratory distress syndrome: the Berlin definition. JAMA 307(23):2526-2533

17. Meade MO, Cook DJ, Guyatt GH, Slutsky AS, Arabi YM, Cooper DJ, Davies AR, Hand LE, Zhou Q, Thabane L, Austin P, Lapinsky S, Baxter A, RussellJ SkrobikY, TE RoncoJJ Stewart (2008) Ventilation strategy using low tidal volume, recruitment maneuvers, and high positive end-expiratory pressure for acute lung injury and acute respiratory distress syndrome: a randomized controlled trial. JAMA 299:637-645

18. Talmor D, Sarge T, Malhotra A, O'Donnell CR, Ritz R, Lisbon A, Novack V, Loring SH (2008) Mechanical ventilation guided by esophageal pressure in acute lung injury. N Engl J Med 359(20):2095-2104

19. Beitler JR, Sands SA, Loring SH, Owens RL, Malhotra A, Spragg RG, Matthay MA, Thompson BT, Talmor D (2016) Quantifying unintended exposure to high tidal volumes from breath stacking dyssynchrony in ARDS: the BREATHE criteria. Intensive Care Med 42(9):1427-1436

20. Habashi NM (2005) Other approaches to open-lung ventilation: airway pressure release ventilation. Crit Care Med 33[3 Suppl]:S228-S240

21. Roy S, Sadowitz B, Andrews P, Gatto LA, Marx W, Ge L, Wang G, Lin X, Dean DA, Kuhn M, Ghosh A, Satalin J, Snyder K, Vodovotz Y, Nieman G, Habashi N (2012) Early stabilizing alveolar ventilation prevents acute respiratory distress syndrome: a novel timing-based ventilatory intervention to avert lung injury. J Trauma Acute Care Surg 73:391-400

22. Brower RG, Lanken PN, Maclntyre N et al (2004) Higher versus lower positive end expiratory pressures in patients with the acute respiratory distress syndrome. N Engl J Med 351:327-336

23. Kollisch-Singule M, Emr B, Jain SV, Andrews P, Satalin J, Liu J, Porcellio E, Kenyon V, Wang G, Marx W, Gatto LA, Nieman GF, Habashi NM (2015) The effects of airway pressure release ventilation on respiratory mechanics in extrapulmonary lung injury. Intensive Care Med Exp 3(1):35

24. Delaunois L (1989) Anatomy and physiology of collateral respiratory pathways. Eur Respir J 2:893-904

25. Bates JH, Irvin CG (2002) Time dependence of recruitment and derecruitment in the lung: a theoretical model. J Appl Physiol 93:705-713

26. Guldner A, Braune A, Carvalho N, Beda A, Zeidler S, Wiedemann B, Wunderlich G, Andreeff M, Uhlig C, Spieth PM, Koch T, Pelosi P, Kotzerke J, de Abreu MG (2014) Higher levels of spontaneous breathing induce lung recruitment and reduce global stress/strain in experimental lung injury. Anesthesiology 120:673-682

27. Neumann P, Wrigge H, Zinserling J, Hinz J, Maripuu E, Andersson LG, Putensen C, Hedenstierna G (2005) Spontaneous breathing affects the spatial ventilation and perfusion. Crit Care Med 33:1090-1095

28. Yoshida T, Roldan R, Beraldo MA, Torsani V, Gomes S, De Santis RR, Costa EL, Tucci MR, Lima RG, Kavanagh BP, Amato MB (2016) Spontaneous effort during mechanical ventilation: maximal injury with less positive endexpiratory pressure. Crit Care Med 44(8):e678-e688

29. Kaplan LJ, Bailey H, Formosa V (2001) Airway pressure release ventilation increases cardiac performance in patients with acute lung injury/adult respiratory distress syndrome. Crit Care 5:221-226

30. Fan E, Khatri P, Mendez-Tellez PA, Shanholtz C, Needham DM (2008) Review of a large clinical series: sedation and analgesia usage with airway pressure release and assist-control ventilation for acute lung injury. J Intensive Care Med 23(6):376-383

31. Blanch L, Villagra A, Sales B, Montanya J, Lucangelo U, Luján M, GarcíaEsquirol O, Chacón E, Estruga A, Oliva JC, Hernández-Abadia A, Albaiceta GM, Fernández-Mondejar E, Fernández R, Lopez-Aguilar J, Villar J, Murias G, Kacmarek RM (2015) Asynchronies during mechanical ventilation are associated with mortality. Intensive Care Med 41(4):633-641

32. McConville JF, Kress JP (2012) Weaning patients from the ventilator. N Engl J Med 367:2233-2239 